\title{
Adaptive Fuzzy Kalman Filter Based on INS/RADAR Integrated Navigation System
}

\author{
Cheng Pengzhan \\ College of Mechanical Engineering \\ Shanghai University of Engineering Science, \\ City Shanghai 201620, China \\ He Fajiang \\ College of Air Transportation and Flying \\ Shanghai University of Engineering Science \\ City Shanghai 201620, China
}

\author{
Dang Shuwen \\ College of Air Transportation and Flying \\ Shanghai University of Engineering Science \\ City Shanghai 201620, China \\ Wang Kangle \\ College of Air Transportation and Flying \\ Shanghai University of Engineering Science \\ City Shanghai 201620, China
}

\begin{abstract}
This paper presents a novel INS/RADAR integrated navigation algorithm based on Fuzzy Adaptive Kalman Filtering. The method is mainly used in INS/RADAR integrated navigation system to deal with time varied statistic of measurement noise in different working conditions. By monitoring the relation between status from INS and status from RADAR, the algorithm recursively modifies the gain coefficients of the filter so as to adaptively control the Kalman filter, and finally the optimal estimation is achieved. Through monitoring RMSE, in the case of similar accuracy, running time of the fuzzy filtering algorithm compared to the traditional Kalman filter algorithm is reduced by $32.73 \%$ the experimentation results of the INS/RADAR integrated navigation system indicate that the algorithm is effective and practical.
\end{abstract}

Keywords-fuzzy logic; integrated navigation; adaptive Kalman filter; Analytic Hierarchy Process

\section{INTRODUCTION}

Kalman filter technology is widely used in the integrated navigation system, which is an effective method for state optimal estimation. When the mathematical model of the complex system is known, the state of the system can be estimated accurately by the conventional Kalman filter. The establishment of a mathematical model requires a lot of testing; especially it is difficult to find out an accurate system noise and statistical characteristics of measurement noise.

A number of previous studies and improvements have been carried out. X.X.Wang ${ }^{[1]}$ has designed an adaptive fusion filtering algorithm based on IMM. Two models are used to describe the system structure, and the Sage-Husa filters corresponding to each model are working with strong tracking filter independently in parallel, then the estimation of the system's state is the weighted fusion result of the model probabilities of the two filtering estimates, with high precision and strong robustness. Through several theoretical studies and attempts, X.R.Li ${ }^{[2]}$ proposed a variable structure multi-model (VSMM), with a time-varying model instead of models with a fixed set; the model set is determined by the adaptive process.
X.S.Shi ${ }^{[3]}$ improves the performance of particle filter by introducing adaptive fuzzy particle filter and changing the size of particle by real-time dynamic, but it is necessary to increase the computational complexity. Y.F.XU ${ }^{[4]}$ updates and forecasts the Fischer information weighting relationship of the state vector and introduces the multiple update algorithm, by which the data fusion is performed on the external information to reduce the linearization error in the measurement update and improve the accuracy of posteriori state estimation, but its calculation depends on the exact work of a single sensor, once the device appears a fixed error, it is difficult to calculate through the iterative calculation. $\mathrm{LI} \mathrm{Xu}^{[5]}$ gives a $\mathrm{H} \infty$ filter, which is actually an extension of Kalman filter, which has no known constraint of the system, but the algorithm is complex and computationally large, so the system requires high hardware.

In this paper, the Kalman filter and the fuzzy logic method are used in combination. According to the real difference between the actual variance and the theoretical variance, the fuzzy system is designed to adjust the confidence of the different sensors in real time. As a result of the introduction of the INS autonomous navigation system, the fusion algorithm does not need to obtain a priori knowledge of the measurement noise matrix, and in the case of any navigation system failure can get accurate estimates.

In practical applications, it is difficult to overcome the divergence problem in traditional Kalman filter: when the new message is no longer white noise, the filter will become unstable, and even the system can't normally output. In this paper, a fuzzy weighted Kalman filter fusion algorithm based on Analytic Hierarchy Process (AHP) is proposed. By dynamically monitoring the variance ratio of the new information, the weights of the data obtained by the different sensors are adjusted adaptively and fuzzily to achieve the purpose of preventing divergence. 


\section{KALMAN FILTER ALGORITHM}

In the Kalman filter algorithm, the state equation and the observation equation can be expressed as:

$$
\begin{aligned}
& X(k+1)=f[k, X(k)]+G(k) W(k) \\
& Z(k)=h[k, X(k)]+V(k)
\end{aligned}
$$

Where, $W(k)$ is system noise, $V(k)$ is measurement noise matrix;

$$
E\{W\}=0, E\{V\}=0
$$

Where, $Q$ is non - negative system noise variance matrix, $R$ is positive measurement of noise system variance matrix.

$$
r=\operatorname{tr}\left[H_{i} P(k+1 / k) H_{i}{ }^{T}+R(k)\right]
$$

Where $r$ is theoretical variance.

\section{DESIGN OF ADAPTIVE FUZZY KALMAN FILTER}

\section{A. Information fusion}

The Joint Kalman filter algorithm is a common method for data fusion in multi-sensor integrated navigation system. It is weighted by the information distribution of each sub-filter to get the global state output. The weights are obtained by synthesizing the sub-filter variance matrix. Considering the complexity of practical application environment, the statistical characteristics of the measurement noise of each subsystem may be changeful. If the sub-filter uses traditional Kalman filter algorithm, the above changes can't be detected, so that the estimation error will increase with these changes. Because the fuzzy adaptive filtering method has good adaptability to the variation of statistical characteristics of measurement noise, this paper proposes a fuzzy adaptive filter instead of traditional Kalman filter in the joint Kalman filter algorithm to form a fuzzy adaptive joint Man information fusion algorithm.

Considering the integrated navigation data fusion system composed of inertial navigation and laser navigation, the inertial navigation is used as the reference system and the laser navigation as the auxiliary sensor. The algorithm of the inertial navigation / laser combination navigation algorithm based on the fuzzy adaptive Kalman fusion algorithm is shown in the following figure:

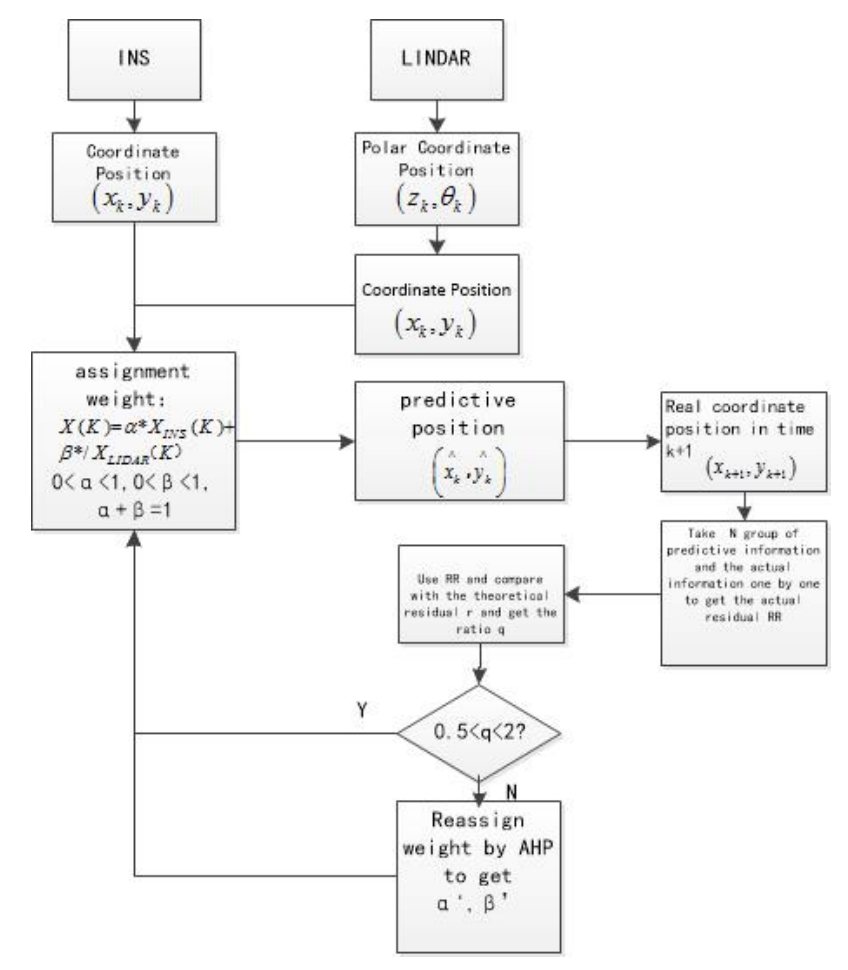

Fig. 1. Flowchart of information fusion

In the initial stage, the initial weights are given according to the experience and the accuracy of the sensors to distribute the weights of the different sensors' data in the algorithm operation:

$$
X 0_{k}=\alpha^{*} X 1_{k}+\beta * X 2_{k}
$$

Where $0<\alpha<1,0<\beta<1, \alpha+\beta=1$.

$X 0_{k}$ replace $X_{k}$ to be gotten into calculation ,on which based we can get the $r$ predictive position $X_{k+1 / k}$.

After calculations are performed in $\mathrm{N}$ times, the calculation is carried out by the following equation:

$$
q(i)=\frac{\operatorname{tr}\left\{\sum_{j=k-N+1}^{N}\left[Z_{j}(k+1)-X_{j}(k+1 / k)\right]\left[Z_{j}(k+1)-X_{j}(k+1 / k)\right]^{T}\right\}}{N r}
$$

$q(i)$ represents the ratio of the measured residual to the theoretical residual $\mathrm{r}$ in $\mathrm{N}$ times calculations. ${ }^{[4]}$

The confidence of each sensor data can be calculated from $q(i)$ obtained by equation (4). The general method is to assume a threshold, if $q(i)$ is greater than this threshold, then the filter threshold, then the filter results are not available at this time. Equivalent to the confidence of only $\{0,1\}$ two values, but this method does not consider the navigator data from available to unavailable The transition process.

When $q(i) \in(1 / 2,2)$, it is assumed that the residual variance is close to the theoretical variance of 1 and the confidence is 1 . When $q(i) \in(0,1 / 10) \cup(10,+\infty)$, the 
residual variance is less than $1 / 10$ or 10 times the theoretical variance, and $q(i)$ is far away from 1 , so the confidence is 0

\section{B. Updating of the proportional relationship by AHP}

In this paper, the position factors $\mathrm{x}, \mathrm{y}$ and the azimuth angle $\theta$ are chosen as the state input. The following model was established by analytic hierarchy process.

Target layer: The accurate and stable operation of the navigation system(A).

Scheme: The residual of the abscissa $x(B 1)$, the residual of the ordinate $y(B 2)$, the residual of the azimuth angle $\theta(B 3)$,

Criterion level: Data from Inertial gyroscope(C1),data from Laser scanner(C2).

We can get the judgment matrix as follow:

\section{1) Judgment matrix $A-C$}

TABLE I. JUDGMENT MATRIX A-C

\begin{tabular}{|c|c|c|}
\hline $\mathrm{A}$ & $\mathrm{C} 1$ & $\mathrm{C} 2$ \\
\hline $\mathrm{C} 1$ & 1 & $\mathrm{a}$ \\
\hline $\mathrm{C} 2$ & $1 / \mathrm{a}$ & 1 \\
\hline
\end{tabular}

The weight of two sensors' data may change after each test, so it is necessary to select an integer a closed to the ratio of the two to describe the weight between the two sensors.

2) Judgment matrix $C 1-B$

TABLE II. JUDGMENT MATRIX C1-B

\begin{tabular}{|c|c|c|c|}
\hline C1 & B1 & B2 & B3 \\
\hline B1 & 1 & 1 & b \\
\hline B2 & 1 & 1 & b \\
\hline B3 & $1 / b$ & $1 / b$ & 1 \\
\hline
\end{tabular}

The gyroscope obtains the position by measuring the acceleration, and the coordinate position $(\mathrm{x}, \mathrm{y})$ is obtained by the same method and same sensor, so their uncertainty can be regarded as the same, $\delta_{x}=\delta_{y}$. The uncertainty of the polar angle $\theta$ in polar coordinates is calculated by the following equation:

$$
\delta_{\theta}=\sqrt{\left(\frac{\partial \theta}{\partial x}\right)^{2} \delta_{x}^{2}+\left(\frac{\partial \theta}{\partial y}\right)^{2} \delta_{y}^{2}}
$$

The uncertainty of the polar angle $\theta$ can be obtained:

$$
\delta_{\theta}=\sqrt{\frac{\delta_{y}^{2} x^{4}+\delta_{x}^{2} y^{2}}{\left(x^{2}+y^{2}\right)^{2}}} .
$$

Because $\delta_{x}=\delta_{y}$, we can get:

$$
b=\frac{\delta_{\theta}}{\delta_{x}}=\frac{\delta_{\theta}}{\delta_{y}}=\sqrt{\frac{x^{4}+y^{2}}{\left(x^{2}+y^{2}\right)^{2}}}
$$

\section{3) Judgment matrix $C 2-B$}

TABLE III. JUDGMENT MATRIX C2-B

\begin{tabular}{|c|c|c|c|}
\hline C2 & B1 & B2 & B3 \\
\hline B1 & 1 & 1 & c \\
\hline B2 & 1 & 1 & c \\
\hline B3 & $1 / \mathrm{c}$ & $1 / \mathrm{c}$ & 1 \\
\hline
\end{tabular}

Output data from radar scanner is $(\mathrm{x}, \mathrm{y}, \theta)$. Simultaneously as above, $\delta_{x}=\delta_{y}, \mathrm{c}=\frac{\delta_{\theta}}{\delta_{x}}=\frac{\delta_{\theta}}{\delta_{y}}=\sqrt{x^{2}+y^{2}} \sin \theta$.

4) Recalculate weights by $A H P$

The calculated new weights are multiplied by the corresponding residuals of the gyroscope data and the laser scanner data, and finally we can get the new ratios $\alpha$ and $\beta$ :

$$
\alpha=\frac{P_{x} * \lambda_{x}^{\text {ins }}+P_{y} * \lambda_{y}^{\text {ins }}+P_{\theta} * \lambda_{\theta}^{\text {ins }}}{P_{x} * \lambda^{\text {ins }}{ }_{x}+P_{y} * \lambda^{\text {ins }}+P_{\theta} * \lambda_{\theta}^{\text {ins }}+P_{x} * \lambda^{\text {lindar }}{ }_{x}+P_{y} * \lambda^{\text {lindar }}{ }_{y}+P_{\theta} * \lambda_{\theta}^{\text {lindar }}}
$$

We can get a new way of data fusion

$$
X 0_{k}=\alpha^{\prime *} X 1_{k}+\beta^{\prime *} X 2_{k}
$$

And update the initial ratio $a$, to achieve adaptive data fusion.

\section{SimULATION AND ANALYSIS}

\section{A. Setting of simulation}

In the experiment, we use both adaptive EKF and traditional EKF to simulate. The robot moves from the coordinates $(0,0)$ and moves counterclockwise as Figure 3 in a $25 * 25$ scale environment. It is positioned according to the laser rangefinder and the inertial navigation system and finally returns to the starting point. Simulation of motion noise and observed noise settings are as follows:

$$
\sigma_{V}=0.3 \mathrm{~m} / \mathrm{s}, \sigma_{r}=3^{\circ}, \sigma_{\rho}=0.1^{\circ}, \sigma_{b}=1^{\circ}
$$

The maximum speed is $90^{\circ}$,the maximum angular velocity is $90^{\circ} / \mathrm{s}$, the maximum scanning distance of the laser scanner is $30 \mathrm{~m}$, the scanning range is $0 \sim 180^{\circ}$, the front and rear wheel spacing is $4 \mathrm{~m}$, the control period is $0.025 \mathrm{~s}$, every 8 control cycles for an observation.

\section{B. Analysis of results}

The integrated navigation system consists of micromechanical inertial and $360^{\circ}$ laser scanners whose state variables can be expressed as:

$$
\mathrm{X}=\left[x, y, \theta, x_{l 1}, y_{l 1}, \theta_{l 1}, \ldots \ldots \ldots x_{\mathrm{ln}}, y_{\mathrm{ln}}, \theta_{\mathrm{ln}}\right] \mathrm{T}
$$


Where $x_{l 1}, y_{l 1}, \theta_{l 1}, \ldots \ldots \ldots x_{l n}, y_{l n}, \theta_{l n}$ is the location of the landmark.

In Figure 2 we use 30 road signs. It is the path of car's movement of the road comparison chart and its partial magnification. Black line, red line, green line, respectively, is on behalf of the real path, the traditional algorithm to estimate the path, fuzzy algorithm to estimate the path. It can be seen that the path planning of the fuzzy algorithm is more accurate than the traditional algorithm's, and with the operation of the algorithm, the traditional algorithm can't adjust the deviation in real time, which leads to not only deviation of the path but also instability.

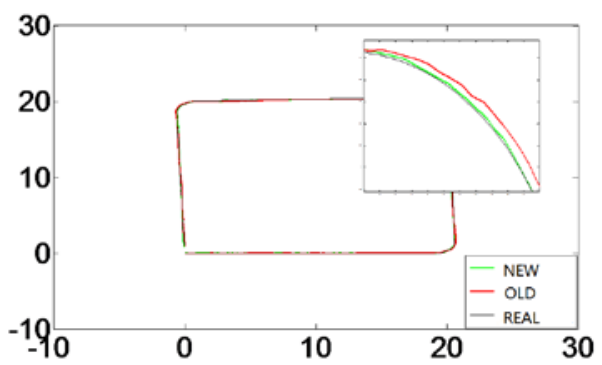

Fig. 2. Path of comparison between traditional Algorithm and new one; enlarged view

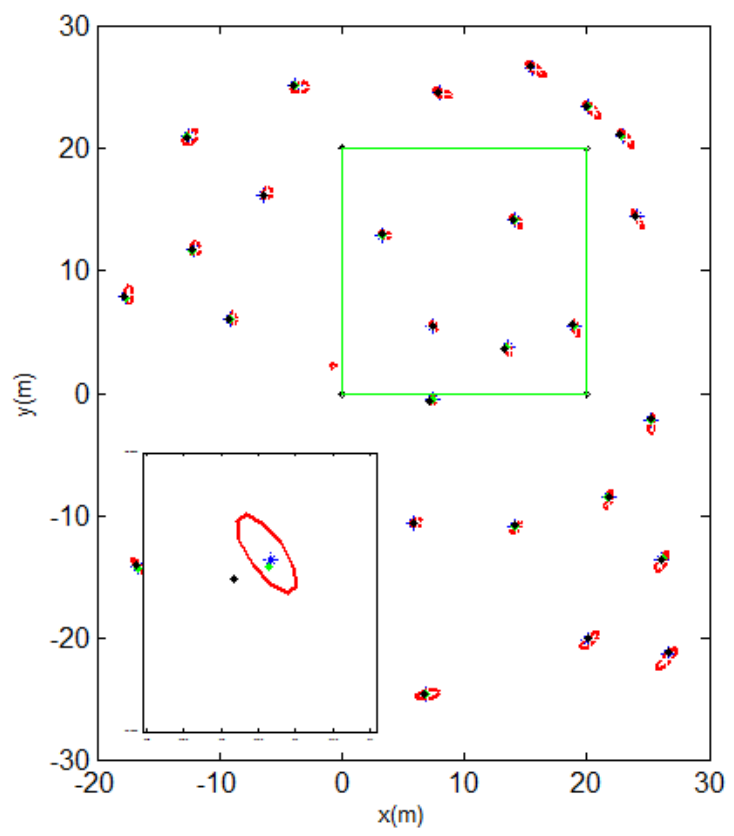

Fig. 3. Comparison of landmarks between traditional Algorithm and new one; enlarged view

Figure 3 compares the map of the landmarks constructed by the two algorithms. The blue dot represents the actual road mark point, and the green point represents the fuzzy algorithm road sign point, and the black dot represents the traditional algorithm; the red ellipse (called the uncertainty ellipse, the confidence degree used here is $97 \%$, Referred to as an indefinite ellipse), indicates the uncertainty of the estimated feature points. It can be obtained from the figure that the landmarks estimated by the fuzzy algorithm are closer to the real signs, and the estimated feature points are located within the ellipse (red).

\section{1) Errors of landmarks}

Figure 4 and Figure 5, respectively, in the number of 50 landmarks, is the absolute error comparison chart of landmarks' $\mathrm{x}$ and $\mathrm{y}$, where abscissa is the number of landmarks to scan. From these figures, when the laser scanner to scan the new road marking point, the increase rate of error increases. The new algorithm distributes the weight by introducing the fuzzy algorithm so that the estimation accuracy of the landmark is much higher than that of the traditional algorithm.

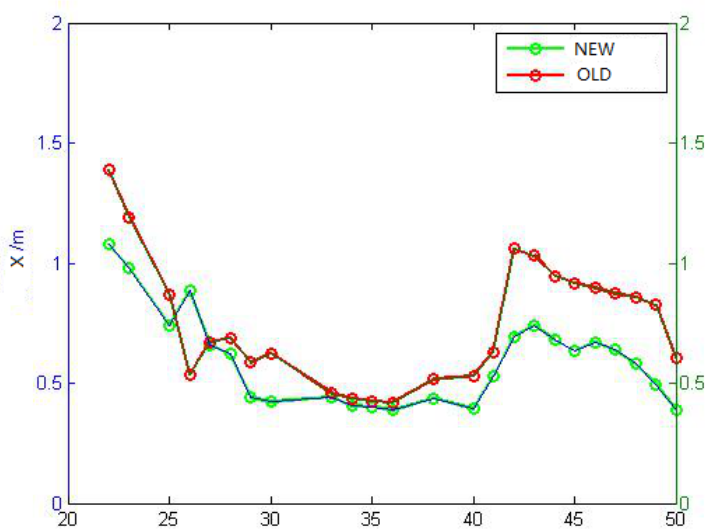

Fig. 4. Absolute error of landmarks' position in $\mathrm{x}$

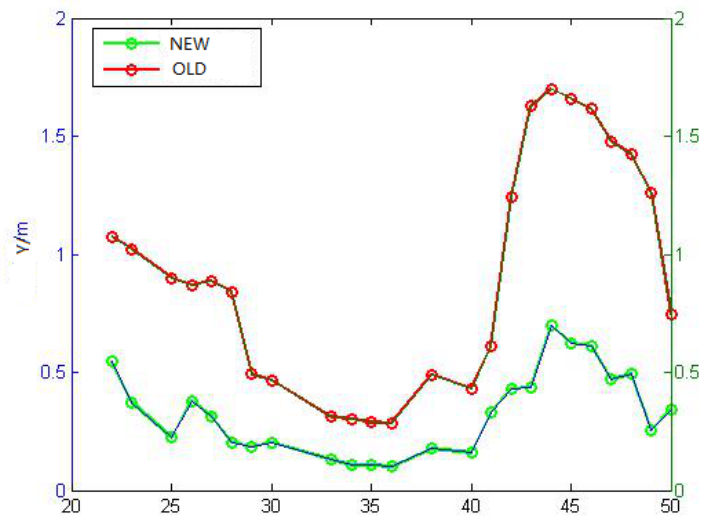

Fig. 5. Absolute error of landmarks' position in y

2) RMSE of the coordinates of the robot position

The robot position coordinates $(\mathrm{x}, \mathrm{y})$ are evaluated by RMSE, and the formula is as follows:

$$
X_{v e}=\sum_{j}^{j=1}\left(X_{f, k}-X_{F, g}\right)^{2}
$$

Where $X_{f, k}$ said the robot measured pose, $X_{F, g}$ said the real posture. 
Table IV shows the RMSE with 10 landmarks, 30 landmarks, 50 landmarks under the two algorithms.

TABLE IV. RMSE OF X AND Y WITH 10,30 AND 50 LANDMARKS

\begin{tabular}{|c|c|c|c|c|c|c|}
\hline \multirow{2}{*}{} & \multicolumn{5}{|c|}{ RMSE } \\
\cline { 2 - 7 } & 10 landmarks & \multicolumn{2}{c|}{30 landmarks } & \multicolumn{2}{c|}{ 50 landmarks } \\
\cline { 2 - 7 } & $\mathrm{x}$ & $\mathrm{y}$ & $\mathrm{x}$ & $\mathrm{y}$ & $\mathrm{x}$ & $\mathrm{y}$ \\
\hline $\begin{array}{c}\text { new } \\
\times 10^{-3}\end{array}$ & 2.733 & 2.640 & 2.489 & 2.519 & 2.324 & 2.266 \\
\hline $\begin{array}{c}\text { traditi } \\
\text { onal } \\
\times 10^{-3}\end{array}$ & 12.47 & 8.976 & 5.733 & 5.798 & 2.677 & 6.012 \\
\hline
\end{tabular}

TABLE V. AVERAGE COMPUTING TIME IN SEVERAL SITUATIONS OF LANDMARK

\begin{tabular}{|c|c|c|c|c|c|c|}
\hline & \multicolumn{2}{|c|}{10 landmarks } & \multicolumn{2}{c|}{30 landmarks } & \multicolumn{2}{c|}{50 landmarks } \\
\hline & New & $\begin{array}{c}\text { Traditi } \\
\text { onal }\end{array}$ & New & $\begin{array}{c}\text { Traditi } \\
\text { onal }\end{array}$ & New & $\begin{array}{c}\text { traditi } \\
\text { onal }\end{array}$ \\
\hline $\begin{array}{c}\text { Ave. } \\
\text { time } \\
\text { cost(s) }\end{array}$ & 14.45 & 8.915 & 22.18 & 14.11 & 30.95 & 26.24 \\
\hline
\end{tabular}

It can be seen from Fig. 4, Fig. 5, and Table IV that the traditional Kalman filter has a residual value higher than that of the new Kalman filter algorithm in the case of less available landmarks, but with the sign number and density Increase, although the root mean square error is reduced, the results are getting closer. As can be seen from Table $\mathrm{V}$, the new algorithm will be more than 3 seconds longer than the traditional algorithm, but with the increase in road signs, the overall timeconsuming growth rate is much larger than the new algorithm more time, at the same time, in the traditional algorithm using 50 The time taken to calculate the road signs is $32.73 \%$ longer than the 30 new road signs.

In conclusion, it can be seen from the experimental results that the filtering result of the new algorithm is superior to the traditional Kalman filter. In the case of fewer landmarks the advantage of new algorithm is more available.

\section{CONCLUSIONS}

In this paper, we design an adaptive fuzzy Kalman filter algorithm which has the AGV car as research object. The results show that:

(1) With same numbers of landmarks in 30, the RMSE of the fuzzy algorithm is reduced by 56.58 compared with the traditional algorithm.

(2) When the traditional algorithm increases the number of landmarks to 50, the time-cost of fuzzy algorithm is reduced by $32.73 \%$ when the new algorithm is in 30 landmarks.

It can be concluded that the information fusion algorithm can obtain the correct weighting value according to the variance of the adaptive detection information, keep the navigation information stationary and accurate, increase the stability of the Kalman fusion algorithm and improve the accuracy of the state estimation State tracking performance.

\section{REFERENCES}

[1] Adaptive Fusion Filtering Algorithm and Its Application for INS / GPS Integrated Navigation System. Journal of Astronautics. 2010,31 (11):2503-2508.

[2] Chidthong Yupa, Tanaka, Hitoshi, Separated Seree. Developing a hybrid multi-model for peak flood forecasting. Hydrological Processes. 2009 ,23(12): 1725-1738.

[3] SHI Xu-sheng, WANG Chen, GUO Li. Application of Adaptive Fuzzy Particle Filter in an Integrated GPS/INS System. Computer simulation,2008,(04):49-52

[4] Y.F.Xu, Z.L.Sun, L.S.Zeng. Mobile Robot SLAM Simulation with Multi-measurement Update. Journal of System Simulation, 2015, (06): 1288-1293

[5] LI Xu, ZHANG Wei-gong. Multi-sensor integrated navigation for intelligent vehicles based on $\mathrm{H} \infty$ filter[J]. Journal of System Simulation, 2007, 19(18): 4168-4177

[6] Tim Bailey, Juan Nieto. Mobile Robot Localization and Mapping in Extensive Outdoor Environments. Australian Centre for Field Robotics, University of Sydney, 2002.

[7] T.L.Xu. Research on Information Fusion Algorithm for Land Vehicle Integrated Navigation and System Development[d]. Harbin Institute of Technology 2007

[8] H.N.Bao. Research of Laser Guided Age Base on Fuzzy Control[D]. Instrument Science and Technology 2016

[9] K.F.Yuan. Key Technique of Multi-source Information Fusion in Integrated Navigation System [D]. Harbin Engineering University 2012.

[10] H.M.M.M. Jayawickramaa, A.K. Kulatungaa, , S. Mathavanb. Fuzzy AHP based Plant Sustainability Evaluation Method .Volume 8, 2017, Pages 571-578

[11] Yunbo Hong,Rongchuan Sun,FRui Lin,et al. Mopping module design and experiments of a multifunction floor cleaning robot[J] . Intelligent Control and Automation (WCICA), 2014 11th World Congress on. 2014,6

[12] Mohamed M. Atia,Shifei Liu,Heba Nematallah. Lidar/Mems Imu integrated navigation (SLAM) method for a small UAV in indoor environments[J].IEEE Transactions on Vehicular Technology.2015, Volume: 64, Issue: 4.Pages: 1279 - 1292.

[13] L.P.Sun. Study on Fuzzy Identification Methods Based on T-S Models and Their Application[D] Yanshan University, 2006

[14] B.G.Li, Q.Ma, S.R.Ding. IEKF Algorithm and Simulation of Passive Location to Moving Target by Single Fixed Observer. Tactical Missile Technology,2012,(05):94-98. 